\title{
Magnetic behavior of lerromagnets with random anisotropy
}

\author{
J. Tejada \\ F. Fisica, Universidad de Barcelona, Diagonal 647, 08028 Barcelona, Spain \\ B. Marinez \\ ICMAB-C.S.I.C., Martí i Franqués S/N, 08028 Barcelona, Spain
}

A. Labarta

F. Física, Universidad de Barcelona, Diagonal 647,08028 Barcelona, Spain

P. Grössinger

Institute For Experimentalphysik, Technical University, Vienna, Austria

\author{
A. Hernando \\ Department de Fisica de Materiales, Universidad Complutense, Madrid, Spain
}

\begin{abstract}
This article reports on a magnetometric study of the effects of diluted local random anisotropy in a ferromagnetic $\mathrm{Fe}_{85} \mathrm{~B}_{20}$ amorphous matrix. In the low-temperature and low-field regime the samples, $F \mathrm{E}_{74} \mathrm{RE}_{6} \mathrm{~B}_{20}(\mathrm{RE}=\mathrm{Nd}$, $\mathrm{Ce})$, show a very rich behavior as a consequence of the competition between, and different dependence on 7 , of the correlation length associated with local randon anisotropy and exchange interactions. In the high-field regime ( $H_{\text {applied }} \geqslant 1.5$ kOe) we observe ferronagnetic behavior with the saturation magnetization varying with temperature according to Bloch's law. The spir wave stiffness constant $D$ could be determined and lies close to $100 \mathrm{meV} \mathrm{A}^{2}$.
\end{abstract}

\section{INTRODUCTION}

Magnetic glasses based on rare earths form an interesting set of systems for studying both phase transitions and magnetization processes in the presence of random interactions. ${ }^{i}$ In these systems we have the competition of random magnetic anisotropy (RMA) and exchange fluctuations due to the existence of random atomic distributions. From the technological point of view these systems have proven to be extremely useful for thermomagnetic recording and magneto-optical readout applications.

An intriguing feature of these systems concens the changes occurring in their magnetic properties when the rare earth atoms are dihted in the iron-boron ferromagnetic matrix. Chuolnovsky and others ${ }^{2,3}$ (CS) have shown that the magnetic structure of such amorphous magnets with ferromagnetic exchange and local random anisotropy (LRA) depends crucially on the parameter $\Lambda_{r} \equiv \lambda_{r}\left(R_{\alpha} / a\right)^{2}$, where $\lambda_{r}$ is the ratio of LRA strength to exchange, $R_{a}$ is the scale of the spatial correlation of the easy axis, and $a$ is the interatomic distance.

In this paper we investigate the macroscopic behavior of the system $\mathrm{Fe}_{74} \mathrm{RE}_{6} \mathrm{~B}_{20}(\mathrm{RE}=\mathrm{Nd}, \mathrm{Ce})$ through detailed studies of the different magnetization processes, $M(H, T)$, at low- and high-magnetic fields between $4.2 \mathrm{~K}$ and room temperature. There are four different parameters that should be taken into account in order to explain the macroscopic behavior of the system. First, we have the ferromagnetic exchange constant $J$ between iron atoms. Due to the structural disorder of amorphous alloys, instead of a unique value there is a distribution of valies for the ferromagnetic exchange constant $\Delta J$. The dilution of the rare earth atoms introduces local random anisotropy axis into the iron-boron ferromagnetic matrix due to its strong magnetocrystalline anisotropy. Finally, we can change the external applied field $H$ to characterize the different states of the system.

\section{EXPERIMENTAL RESULTS AND DISCUSSION}

A set of ribbons of composition $\mathrm{Fe}_{74} \mathrm{RE}_{6} \mathrm{~B}_{20}$ ( $\mathrm{RE}=\mathrm{Nd}$ and $\mathrm{Ce}$ ) were prepared by the single roller technique. Detailed description of the growth process and samples can be found eisewhere. ${ }^{4}$ Magnetic measurements were carried out by using a SQULD magnetometer. In Fig. 1 we present the magnetization of the samples at $4.2 \mathrm{~K}$ as a function of the applied magnetic field. The demagnetizing process from saturation has been studied by varying the temperature between $4.2 \mathrm{~K}$ and room temperature keeping constant the inplane applied field of $1 \mathrm{~T}$ which is enough to saturate the sampies. According to conventional spin-wave theory the thermal variation of the magnetization is given by the following expression:

$$
M(T) / M(0)=1-B T^{3 / 2}-C T^{5 / 2},
$$

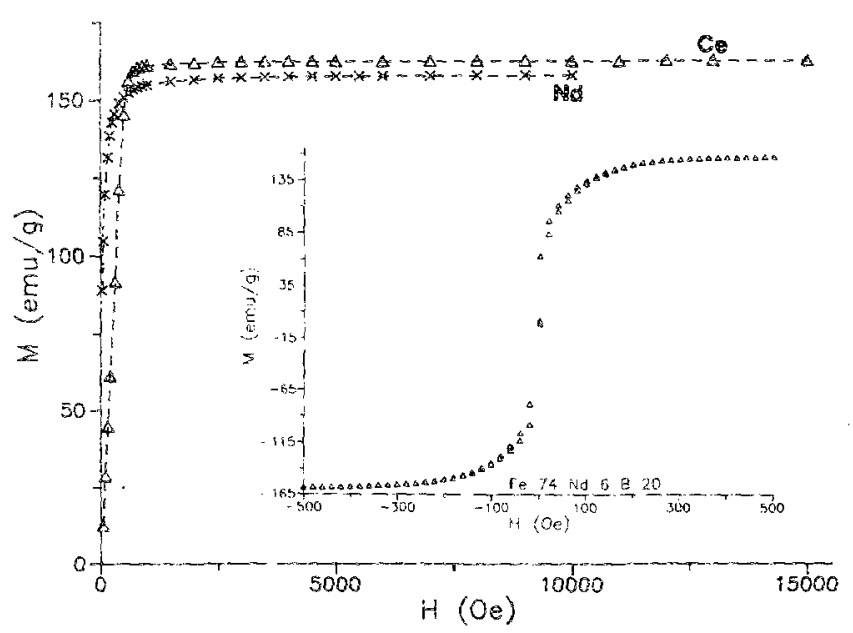

FIG. 1. Isothermal magnetization curves at $T==4.2 \mathrm{~K}$. The inset shows the hysteresis loop at $4.2 \mathrm{~K}$ for the Nd sample. 
where $B$ is related to the spin-wave stiffness constant $D$ through $B=2.62 \mathrm{~g}\left[\mu_{B} / M(0)\right]\left(K_{B} T / 4 \pi D\right)^{3 / 2}$.

Our observed saturation magnetization $M(T)$ obeys such a $T^{3 / 2}$ law. In Fig. 2 we show the experimental $M(T)$ curves and the corresponding fits obtained by using Eq. (1) and taking into account the effects of the applied field. It is evident from Fig. 2 that the behavior of the systern is in excellent agreement with Eq. (1) and only in the very-lowtemperature regime do the data points reffect some differences, suggesting a much more complicated behavior due to the existence of local random anisotropy in the samples. The values obtained from the fits for the spin-wave stiffness constant $D$ are close to $100 \mathrm{meV} \AA^{2}$, which are typical for this kind of material. ${ }^{5}$ Further, the value of $M(0)$ indicates a ferromagnetic coupling between RE and Fe (Ref. 6) atoms.

Initial magnetization curves and hysteresis loops do not show coercive properties, as was predicted by Chudnovsky and Serota ${ }^{2}$ for the correlated spin glass state (CSG). This is characterized by a smooth rotation of the magnetization over the total volume of the sample so that the directions of the magnetization are ferromagnetically correlated within regions of size $R_{D}$ much greater than the interatomic distance. The main macroscopic features of a correlated spin glass are the reversible magnetization curve and the large zero-field susceptibility, $x \cong \Lambda_{r}^{-4}$. We have also carried out low-field magnetization measurements, obtaining the results that we show in Fig. 3. To provide a frame work for the discussion of the magnetic properties of our samples, we consider the model described by CS. Following their notation, we can characterize the different states of our samples as we increase magnetic applied field and/or temperature.

When the random anisotropy is large $\left(H_{r}>H_{\mathrm{FXX}}\right)$ each spin is directed almost along the local random anisotropy axis. The magnetic susceptibility then is very small and a very large magnetic field is needed to reach a reorientation of the spins in the hemisphere defined by the field. In this case the system exhibits a finite coercivity and hysteretic behavior. ${ }^{\text {? }}$

When the random anisotropy is weak $\left(H_{r}<H_{E X}\right)$, as is our case, the system retains some aspects of its collective

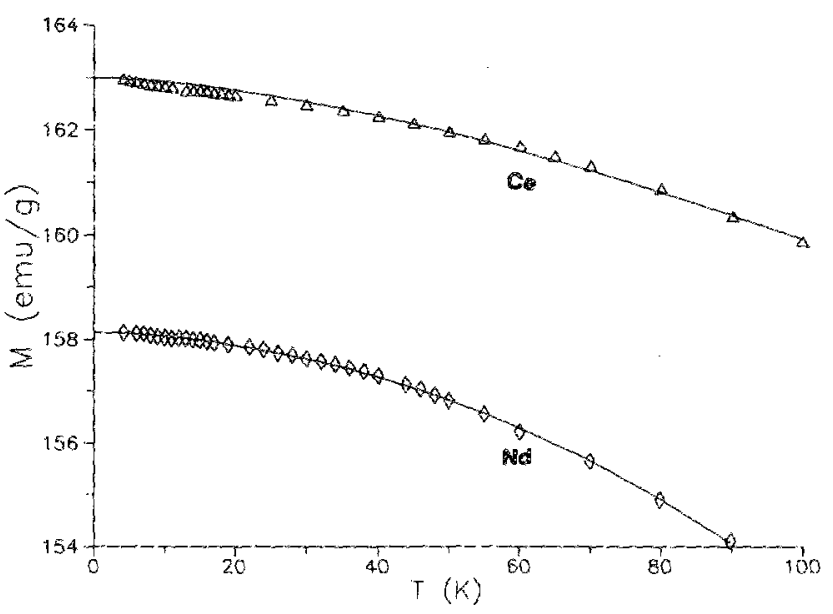

FIG. 2. Variation of the saturation magnetization with temperature at constant applied field $H=1.5 \mathrm{kOe}$.
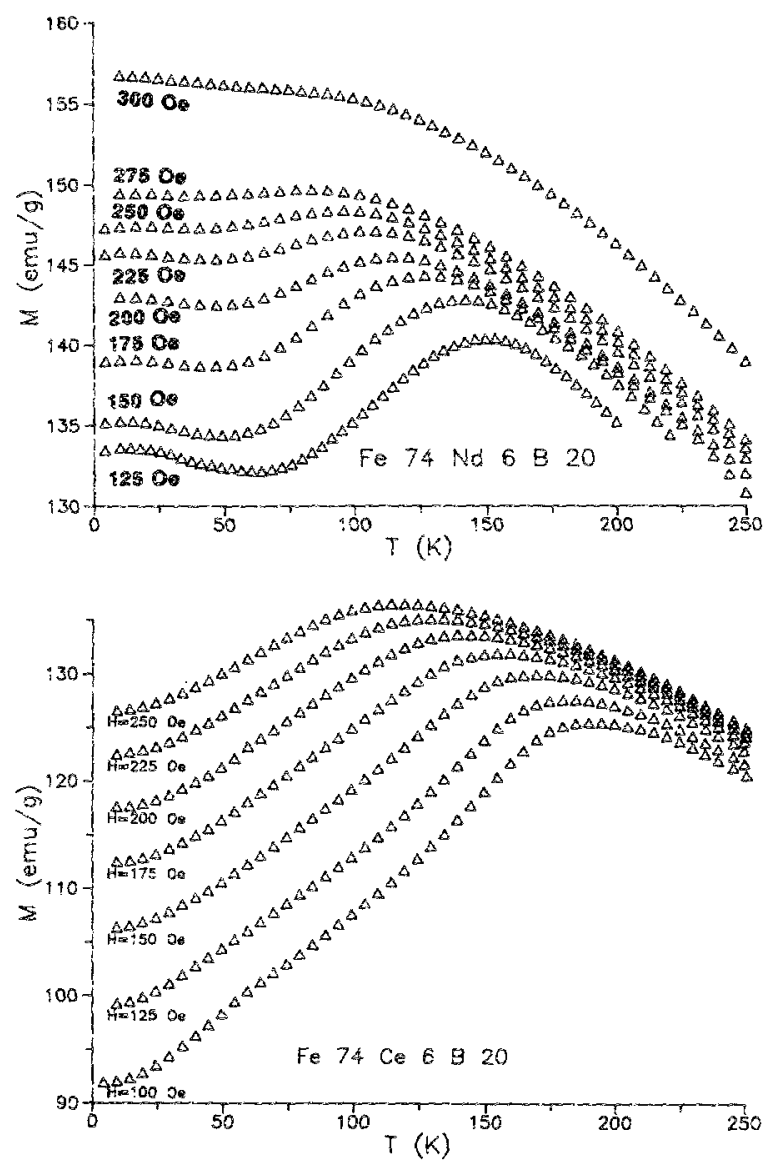

FIG. 3. Magnetization vs temperature for the two samples in the low applied field regime.
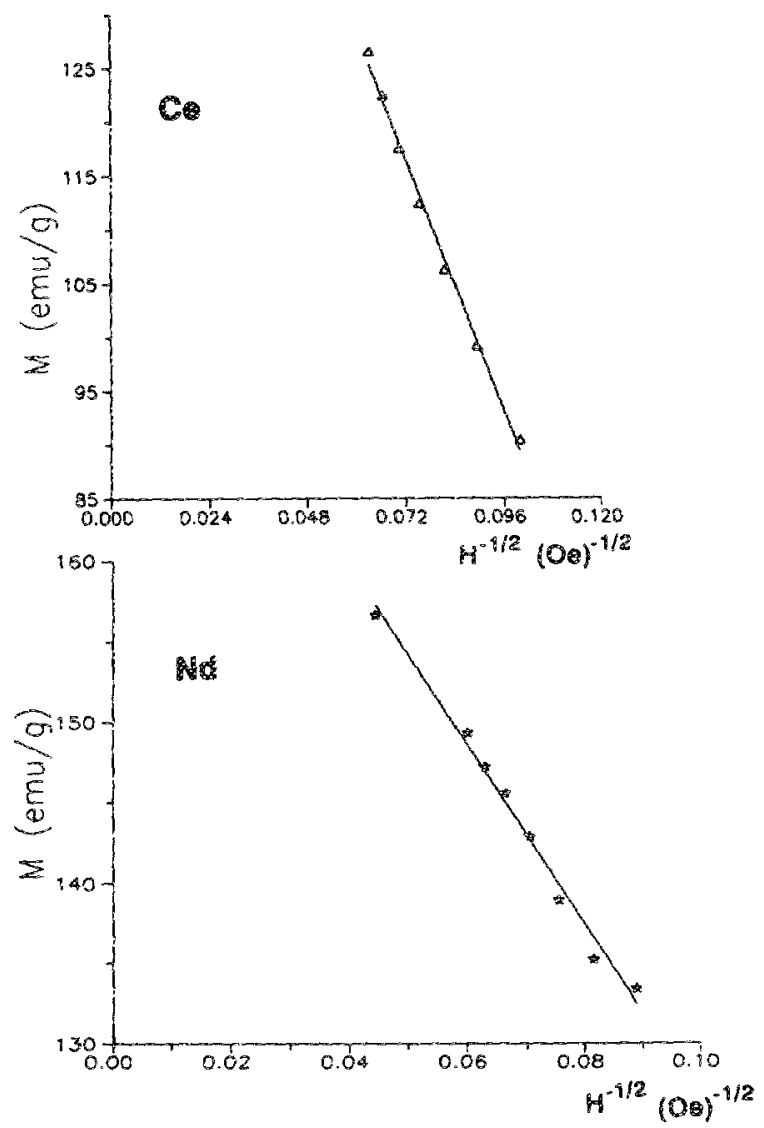

FIG. 4. Magnetization dependence on $H^{-1 / 2}$. Solid lines correspond to the straight line fit. $T=4.2 \mathbb{K}$. 
behavior when no random anisotropy is present. As we have mentioned above, this state is called a correlated spin glass and its main macroscopic features are the absence of hysteresis and the large zero-field susceptibility. The characteristic correlation length over which the spins retain local ferromagnetic order is given by ${ }^{3} R_{\alpha}\left(H_{E X} / H_{R}\right)^{2}$. When an external field $H$ is applied, an alignment of the spins cocurs and, if the field is strong enough ( $H>H_{s} \equiv H_{r}^{4} / H_{\mathrm{EX}}^{3}$ ), it aligns the CSG, leading to a new state that CS called a ferromagnet with a wandering axis. This is a slightly noncollinear structure in which the deviation of the magnetization relative to the field changes with position over the system.

In the correlated spin glass state the system is characterized by an approach to saturation which varies as $H^{-1 / 2}$. In Fig. 4 we show the variation of the magnetization with the applied field and it shows a very good agreement with a $H^{-1 / 2}$ law in the low applied field regime. As we increase the applied field, the field dependence of the magnetization changes to a $H^{-2}$ law in good agreement with CS predictions.
A similar crossover from the CSG to ferromagnetic state can be induced by increasing the temperature of the sample. It is well known that crystalline anisotropy can depend on high powers of magnetization ${ }^{8}$; therefore, a slight decrease of magnetization with increasing temperature might result in a sharp decrease of the random anisotropy strength. ${ }^{3}$

With increasing $T$, the CSG might be transformed into an ordinary ferromagnet due to its large ferromagnetic correlation length, which increases as $T$ increases.

${ }^{1}$ D. J. Sellmyer and S. Nafis, J. Appl. Phys. 57, 3584 (1985).

${ }^{2}$ E. M. Chudnovsky and R. A. Serota, J. Phys. C 16, 4181 (1983).

${ }^{3}$ E. M. Chudnowsky, W. M. Saslow, and R. A. Serota, Phys. Rev. B 33, 251 (1986).

${ }^{4}$ R. Grössinger, H. Sassik, R. Wezulek, and T. Tarnoczi, I. Phys. (Paris) Collog. 49, C8-1337 (1988).

${ }^{5}$ H. Kronmüller and H. Grimn, J. Magn. Magn. Mater. 6, 57 (1977).

${ }^{6}$ R. Grössinger, H. Sassik, R. Wezulek, A. Hernando, G. Rivero, M. Vazquez, B. Martinez, A. Labarta, and J. Tejada (to be published).

'E. Callen, Y. Liu, and J. R. Cullen. Phys, Rev. B 16, 2673 (1977).

${ }^{2}$ H. B. Callen and E. Callen, J. Phys. Chem. Solids 27, 1271 (1966). 